\title{
Evaluación y validación diagnóstica de las variables iQT, razón iQTRR, razón iRTRR para el diagnóstico DEQTc prolongado.
}

\author{
Claudio Santibáñez ${ }^{1}$, Josep Brugada ${ }^{2}$, Paula Soto-Parada ${ }^{a}$, María Santibáñez ${ }^{b}$. \\ 1. Centro de Salud Cardiovascular, SS Puerto Montt, Chile. \\ 2. Hospital Clínic Barcelona España. \\ a. Enfermera Universidad Austral, Sede Puerto Montt, Chile. \\ b. Alumno de Medicina Universidad Finis Terrae, Santiago de Chile.
}

(Financiamiento propio)

Antecedentes: El intervalo QT representa la despolarización y repolarización ventricular y su prolongación está asociada a un mayor riesgo de arritmias graves y muerte súbita. Depende de la frecuencia cardíaca y su rápida valoración es difícil de obtener en la práctica clínica. Una forma que facilita este proceso es medir sólo el intervalo QT pero este no siempre se relaciona con un intervalo QTc prolongado. Debido a esto, se postula que una variable compuesta debería tener mejor rendimiento diagnóstico para este objetivo.

Objetivo: Describir la correlación de 3 variables electrocardiográficas; intervalo QT (iQT), razón del intervalo QT intervalo RR (\%iQTRR) y razón del intervalo RT intervalo RR (\%iRTRR), en relación a la variable QT corregido (iQTc). Se efectuó una evaluación diagnóstica y validación de las variables mencionadas para establecer el diagnóstico de intervalo QT largo y las propiedades diagnósticas de las mismas para el diagnóstico de iQTc prolongado según la fórmula Bazett.

Métodos y Resultados: Se efectuó un estudio transversal descriptivo-relacional, usando una muestra no probabilística formada por 220 elec- trocardiogramas registrados en la base de datos del centro cardiovascular. Las mediciones provenían de ECGs registrados en reposo con medición con cáliper de los intervalos QT y RR en derivadas D2 y/o V5, expresadas en milisegundos. Se excluyó la presencia de isquemia, preexcitación y repolarización precoz. Se obtuvo como resultado que la variable razón iQTRR tiene mejor correlación, concordancia, sensibilidad y valor predictivo negativo para el diagnóstico del iQTc prolongado, versus las variables iQT y razón iRTRR, principalmente en mujeres.

Conclusiones: La variable razón iQTRR tiene una mejor correlación, concordancia, sensibilidad y valor predictivo negativo (VPN) para el diagnóstico del iQTc prolongado, comparado con las variables iQT y razón iRTRR, principalmente, en mujeres. Utilizando ambas variables este efecto se potencia y permite concluir que si una mujer tiene un iQT observado $<470 \mathrm{~ms}$, y una razón QTRR< $47,5 \%$, esto se corresponde con un intervalo QTc normal, con un VPN de $100 \%$.

Palabras clave: Síndrome QT largo, electrocardiograma, QT prolongado, arritmia cardíaca. 


\section{Validation of iQT, iQTRR, iRT/RR ratio for estimation of QTc}

Background: The QT interval (QT) includes depolarization and repolarization phases of cardiac cycle. Its prolongation is associated to an increased risk of serious arrhythmia and sudden death. QT depends on heart rate and it is usually corrected using the Bazett formula (QTc). Prolonged QT is not usually well related to QTc. We postulate that a composite formula muy have a greater value for predicting serious arrhythmia.

Aim: To correlate the predictive value of a different approach to estimate a corrected QT interval: the ratio of RT to RR intervals (QT/RR, expressed as a percentage
Methods and Results: A non probabilistic sample of 220 ECGs were obtained from our data base. ECGs were recorded at rest and measurements were performed in leads Ds and/or V5 using zetcalipers, the values being expressed in $\mathrm{ms}$. The presence of ischemia, pre excitation and early repolarization were excluded. The QT/RR ratio showed better correlation, concordance, sensitivity, and negative predicted value with the usual QTc interval compared to QT alone or de RT/RR ratio, especially so in women.

Keywords: long QT syndrome; electrocardiogram; QT prolonged; arrhythmia cardiac. 


\section{Introducción:}

La medida del iQT en el electrocardiograma (ECG) de reposo es considerada como la representación sumada de la despolarización y repolarización ventricular ${ }^{1}$. La medición de este intervalo requiere al menos 2 derivaciones que muestren de manera clara la onda $\mathrm{T}$ (que se determina mejor en las derivaciones de D11, V2, V5 y V6). Se recomienda que la medición sea manual ${ }^{2,3}$. Por tal motivo, en el presente estudio se utilizaron las derivadas DII y/o V5 medidas de manera manual.

El iQT guarda una relación directa con el intervalo RR $(\mathrm{iRR})^{4}$. Por ello, se han diseñado fórmulas matemáticas para "corregir" el iQT y adecuarlo a la frecuencia cardíaca. El iQT clínicamente útil es el QT corregido (QTc). Aunque no existe un estándar de oro para su diagnóstico, existen varias fórmulas para corregir el iQT; la más empleada es la de Bazett descrita en $1920^{5}$.

Por otro lado, el iQT no sólo depende del iRR precedente sino que también se adapta a los cambios de la frecuencia cardíaca. Entonces, la exactitud del diagnóstico clínico es clave en el Síndrome QT largo, patología de muy baja prevalencia, pero que puede ser mortal6 y para el cual existe un tratamiento eficaz ${ }^{7,8}$. Aparte del ECG, las pruebas diagnósticas de test de esfuerzo y ECG-Holter de 24 horas $^{9}$, son las mejores formas de evaluar la dinámica del iQT día/noche y la influencia del sistema nervioso autónomo en el corazón. Dada la baja prevalencia iQT prolongado se considera importante contar con pruebas diagnósticas más prácticas, eficientes y con un valor predictivo negativo (VPN) superior a las actuales para establecer un diagnóstico preciso. Existe literatura que apoya la observación de la razón $\mathrm{QT} / \mathrm{RR}>50 \%$ se relaciona a un iQTc prolongado, pero no hay descripción de sus propiedades diagnósticas.

El objetivo de este trabajo fue describir las propiedades diagnósticas de 3 variables electrocardiográficas de uso habitual: intervalo QT (iQT), razón del iQT con iRR (\%iQTRR), razón del intervalo RT del iRR (\%iRTRR) y contrastarlos con la variable intervalo (iQTc) prolongado $^{10}$ (Figura 1).

\section{Métodos:}

Se realizó un estudio transversal descriptivo-relacional, con muestreo no probabilístico. Se analizaron registros de 220 ECG tomados en reposo, obtenidos de la base de datos del Centro Cardiológico SS Puerto Montt en el período comprendido entre junio 2014 y

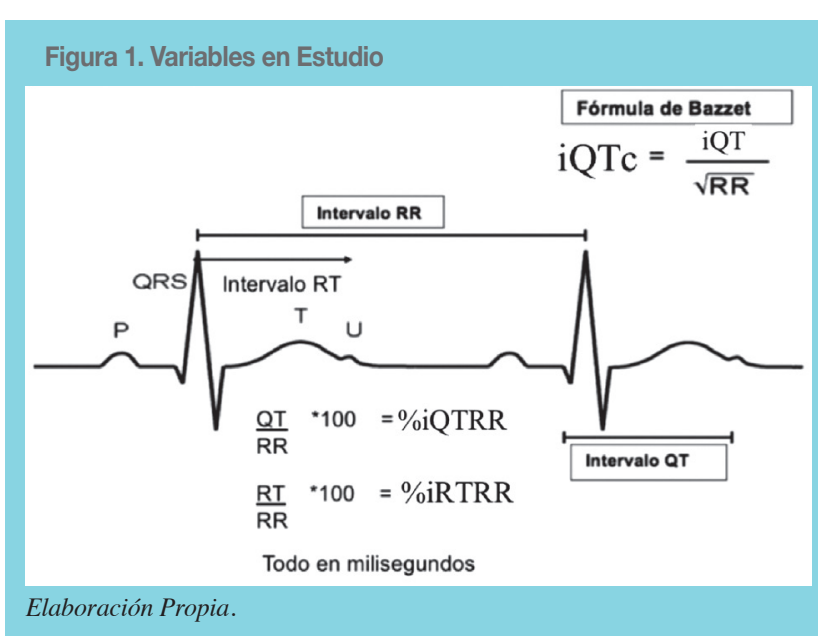

mayo 2016. Por cada ECG con valor del iQTc prolongado (Varón, valor $>450 \mathrm{~ms}$ o mujer $>470 \mathrm{~ms}$ ) se eligió un ECG con valor de iQTc normal ${ }^{10}$. Se excluyen los ECG de pacientes con isquemia, preexcitación, ritmo de marcapasos y repolarización precoz.

Las mediciones fueron manuales por tres operadores independientes previamente entrenados y los valores obtenidos fueron promediados. La calibración del ECG en velocidad y amplitud fue la estándar; la medición fue hecha con cáliper, la unidad de medición fue en milisegundos y la medición fue en la derivada D2 y/o V5, durante 3 ciclos cardíacos, incluyendo la onda U. Ante la presencia de fibrilación atrial se tomaron 10 ciclos. Se usó como gold estándar para el diagnóstico de QT prolongado la fórmula de Bazett dada su amplia difusión, a pesar de sus conocidas limitaciones que tienden a sobre corregir el valor del QT con valores altos de frecuencia cardíaca. Además, se registró edad, género, frecuencia cardíaca, tipo de ritmo, trastornos de conducción intraventricular (bloqueo completo de rama izquierda (BCRI) y derecha (BCRD)) IPR, iRT, iQT, iQTc, iRR, \%iQTRR y \%iRTRR. El análisis estadístico se efectuó usando SPSS-25, evaluando normalidad y homogeneidad de varianza (Prueba de K-S y Levene), correlación de Spearman y concordancia con índice de Kappa, con un nivel de significancia de 0,05. Las variables se dicotomizaron según punto establecido por curva ROC para el diagnóstico de iQTc prolongado (Varón $>450 \mathrm{~ms}$ y mujer $>470 \mathrm{~ms}$ ). El valor iQT prolongado (Varón $>430 \mathrm{~ms}$ y mujer $>470 \mathrm{~ms}$ y la razón QTRR (Varón $>49,5 \%$ y mujer $>47,5 \%$ ) considerando una significancia estadística de $<0,05$. Se calculó sensibilidad, especificidad, valor predictivo positivo (VPP), valor predictivo negativo (VPN) y se estableció concordancia con el índice Kappa. 
Tabla 1. Descripción de la población.

\begin{tabular}{|c|c|c|c|c|c|}
\hline Variables & media & Valor minimo & Valor máximo & Desv. Estándar & total \\
\hline Genero masculino(\%) & 57 & & & & 220 \\
\hline Ritmo Sinusal(\%) & 90 & & & & 220 \\
\hline Bloqueo completo rama izquierda(\%) & 18 & & & & 220 \\
\hline Bloqueo completo rama derecha(\%) & 14 & & & & 220 \\
\hline Intervalo QRS>100 MS(\%) & 43 & & & & 220 \\
\hline $\operatorname{TCIV}(\%)$ & 51 & & & & 220 \\
\hline Intervalo QTc largo corregido por sexo(\% & 50 & & & & 220 \\
\hline Edad (años) & 59,12 & 14 & 96 & 18,79 & 220 \\
\hline Fcia. cardiaca (Ipm) & 76,8 & 47 & 150 & 20,1 & 220 \\
\hline Intervalo PR (ms) & 137,1 & 0 & 300 & 61,6 & 220 \\
\hline Intervalo RR (ms) & 828 & 400 & 1277 & 190,4 & 220 \\
\hline Intervalo QT (ms) & 418 & 260 & 720 & 83,8 & 220 \\
\hline Intervalo RT (ms) & 363,5 & 200 & 680 & 76,5 & 220 \\
\hline Intervalo QRS (ms) & 96,8 & 40 & 300 & 39,3 & 220 \\
\hline Intervalo QTc (ms) & 464 & 284 & 760 & 82,37 & 220 \\
\hline Razon QTRR (\%) & 52,4 & 31 & 93 & 12,54 & 220 \\
\hline Razon RTRR(\%) & 45 & 24 & 80 & 11,38 & 220 \\
\hline
\end{tabular}

\section{Resultados:}

La Tabla 1 muestra las variables descriptivas de la población. La prevalencia de intervalo QTc prolongado fue de 50\%, edad promedio 59 años, el $57 \%$ fueron varones, la frecuencia cardíaca media de $76 \mathrm{lpm}$, el promedio del iQT observado fue de $418 \mathrm{~ms}$ y del iQTc 464 ms; el $51 \%$ presentó un trastorno de conducción intraventricular, de los cuales el $18 \%$ fue por BCRI, y el $14 \%$ por BCRD. No hubo arritmias ventriculares, $y$ un $10 \%$ tuvo fibrilación atrial. El valor promedio de la variable razón iQTRR fue de $52,4 \%$, y de la variable razón iRTRR $45 \%$.

Se observó una correlación significativa de las variables razón iQTRR con razón iRTRR, principalmen-

Tabla 2: Comparación de medias y asociación estadística según diagnóstico de QTc prolongado V>450, M>470.

\begin{tabular}{|c|c|c|c|c|c|c|c|}
\hline Variable & QTc Normal & & & QTc prolongado & & & valor de $\mathrm{p}$ \\
\hline Genero masculino(\%) & 62 & & & 53 & & & 0 \\
\hline Ritmo Sinusal(\%) & 95 & & & 84 & & & 0,04 \\
\hline Bloqueo completo rama izquierda(\%) & 4 & & & 32 & & & 0 \\
\hline Bloqueo completo rama derecha(\%) & 10 & & & 17 & & & 0,117 \\
\hline $\operatorname{TCIV}(\%)$ & 37 & Valor minimo & Valor máximo & 65 & Valor minimo & Valor máximo & $\mathbf{0}$ \\
\hline Edad (años) & 54,3 & 14 & 96 & 63,9 & 19 & 92 & 0,21 \\
\hline Fcia. cardiaca (Ipm) & 71,9 & 47 & 143 & 81,72 & 48 & 150 & 0,001 \\
\hline Intervalo PR (ms) & 133,82 & 0 & 240 & 140,36 & 0 & 300 & 0 \\
\hline Intervalo RR (ms) & 865,6 & 420 & 1277 & 791 & 400 & 1250 & 0,001 \\
\hline Intervalo QT (ms) & 374 & 260 & 480 & 463 & 300 & 720 & 0 \\
\hline Intervalo RT (ms) & 330,5 & 200 & 440 & 396,5 & 260 & 680 & 0,01 \\
\hline Intervalo QRS (ms) & 81,8 & 40 & 160 & 112,4 & 44 & 360 & 0 \\
\hline Intervalo QTc (ms) & 403,8 & 284 & 451 & 525,8 & 450 & 760 & $\mathbf{0}$ \\
\hline Razon QTRR (\%) & 44,05 & 31 & 67 & 60,9 & 42 & 93 & 0 \\
\hline Razon RTRR(\%) & 38,9 & 24 & 76 & 52,09 & 30 & 80 & 0 \\
\hline
\end{tabular}


Tabla 3: Propiedades diagnósticas de las variables en estudio para diagnóstico de QTc prolongado*.

\begin{tabular}{|l|c|c|c|c|c|c|c|c|}
\cline { 2 - 11 } \multicolumn{1}{c|}{} & \multicolumn{2}{c|}{ iQT ** } & \multicolumn{2}{c|}{ Razon QT/RR *** } & QT y/o Razon QT/RR QT y Razon QT/RR \\
\hline \multicolumn{1}{c|}{ Propiedades } & Varon & Mujer & Varon & Mujer & Varon & Mujer & Varon & Mujer \\
\hline Sensibilidad & 62 & $\mathbf{7 5}$ & $\mathbf{8 8}$ & $\mathbf{9 0}$ & $\mathbf{1 0 0}$ & $\mathbf{1 0 0}$ & 50 & 65 \\
\hline Especificidad & $\mathbf{9 0}$ & $\mathbf{9 3}$ & 78 & $\mathbf{7 9}$ & 68 & $\mathbf{7 4}$ & $\mathbf{1 0 0}$ & $\mathbf{9 8}$ \\
\hline Valor predictivo(+) & $\mathbf{8 4}$ & $\mathbf{9 3}$ & $\mathbf{7 7}$ & $\mathbf{8 4}$ & $\mathbf{7 3}$ & $\mathbf{8 3}$ & $\mathbf{1 0 0}$ & $\mathbf{9 7}$ \\
\hline Valor predictivo (-) & $\mathbf{7 3}$ & $\mathbf{7 5}$ & $\mathbf{8 8}$ & $\mathbf{8 7}$ & $\mathbf{1 0 0}$ & $\mathbf{1 0 0}$ & $\mathbf{7 0}$ & 69 \\
\hline Concordancia & 54 & 47 & 65,7 & 69,6 & 70 & 78,1 & 59 & 64,5 \\
\hline KAPPA & 54,5 & 66 & 65,7 & 69,9 & 70 & 75,7 & 59 & 60,5 \\
\hline
\end{tabular}

*iQTc prolongado $>450 \mathrm{~ms}$ varones y $>470 \mathrm{~ms}$ mujeres

te, en el grupo de mujeres. La variable razón iQTRR mostró una correlación de $85 \%$ versus $82 \%$ con la medición clásica del iQT, para diagnóstico de iQTc prolongado. La Tabla 2, muestra la comparación de medias según la presencia de QTc prolongado, y existe diferencia estadísticamente significativa para el tipo de ritmo, intervalo $\mathrm{PR}$, intervalo QRS y para la presencia de BCRI.

Se informó sensibilidad, especificidad, valor predictivo positivo (VPP), VPN y se estableció concordancia con el índice Kappa. Para la variable iQT varón $>430$ ms se obtuvo una sensibilidad $62 \%$, especificidad $90 \%$, VPP $84 \%$ y VPN $73 \%$, concordancia de $54 \%$ e índice kappa de 54,5. En cambio, para la variable razón iQTRR varón $>49,5 \%$ se obtuvo una sensibilidad de $88 \%$, especificidad de $78 \%$, VPP $77 \%$ y VPN de $88 \%$ y concordancia e índice kapa de $65,7 \%$ para el diagnóstico del iQTc prolongado (Tabla 3) Estos valores son superiores en la mujer.

También se describe que al utilizar las dos variables se puede alcanzar un $100 \%$ de sensibilidad, especificidad, VPP, VPN, con una concordancia de $78 \%$ e índice Kappa de 75,7.

Dicho de otro modo, se demostró que en esta población la variable razón iQTRR tiene una mejor correlación, concordancia, sensibilidad y VPN para el diagnóstico del iQTc prolongado, comparado con las variables iQT y razón iRTRR, principalmente en mujeres.

Utilizando ambas variables este efecto se potencia y permite concluir que si una mujer tiene un iQT observado $<470 \mathrm{~ms}$, y una razón QTRR $<47,5 \%$, estaremos frente a un intervalo QTc normal, con un VPN de $100 \%$.

\section{Conclusión y discusión:}

El intervalo QT incluye los tiempos de la despolarización y la repolarización ventricular. Mientras mayor sea su duración aumenta el riesgo de arritmias ventriculares y muerte súbita. La prolongación aparece en distintos tipos de patologías, congénitas o adquiridas, consumo de algunos fármacos como amiodarona, macrólidos o el trióxido de arsénico; este último causa prolongación de iQT en el $80 \%$ de los paciente que lo reciben 11 .

En el diagnóstico, se utiliza la fórmula de Bazett, que consiste en corregir el iQT según la frecuencia cardíaca, medido en milisegundos a través de su fórmula, ${ }^{5}$ lo cual tiene limitaciones descritas por sobre corregir los valores a frecuencias altas. En nuestro estudio la dispersión de frecuencia cardíaca fue entre 47 y 150 $1 \mathrm{pm}$ lo cual puede influir en los resultados. Hubo diferencias asociadas a la presencia de fibrilación atrial, probablemente por mayor frecuencia cardíaca asociada. Solo el BCRI, y no el BCRD, produjo diferencias. Otra limitación es la variabilidad Inter observador que no fue registrada, así como tampoco se registró el consumo de fármacos. Dada la ausencia de eventos arritmogénicos de importancia y la alta prevalencia de QT prolongado en esta población, ella sea de bajo riesgo. En este estudio se describe una herramienta diagnóstica fácil de usar, se demuestra que la variable razón iQTRR tiene una mejor correlación, concordancia, sensibilidad para el diagnóstico del iQTc prolongado y en especial el VPN que alcanza casi a un $90 \%$ y aún mayor cuando se usan en conjunto. La gran ventaja diagnóstica en las patologías de baja prevalencia es 
precisamente el VPN. De esta forma, podemos rápidamente tamizar la lectura electrocardiográfica y aplicar la fórmula de Bazett, sólo en los electrocardiogramas que muestren una razón $\mathrm{QT} / \mathrm{RR}>50 \%$, ya que en todos los demás, la probabilidad de tener un valor normal es cercano al $90 \%$ y si se suma el valor del QT observado,

\section{Referencias}

1.- LOCATI E, BAGLIANI G, PADELETTI L. Normal ventricular repolarization and QT interval: Ionic background, modifiers, and measurements. Card Electrophysiol Clin. 2017; 9 (3): 487 - 513. Disponible en https://pubmed.ncbi.nlm.nih. gov/28838552/?from_single_result=Card+Electrophysiol+Clin.+2017\%3B9\%283\%29\%3A487-513 (consultado el 10 de junio 2020).

2.- POSTEMA P, WILDE A. The Measurement of the QT Interval. Curr Cardiol Rev. 2014; 10 (3): 287 - 294. Disponible en https://www.ncbi.nlm.nih.gov/pmc/articles/PMC4040880/ (consultado el 10 de junio 2020).

3.- VETTER V. Clues or Miscues? How to make the right interpretation and correctly diagnose long-QT syndrome. Circulation. 2007; 115 (20): 2595 - 2598. Disponible en https://pubmed. ncbi.nlm.nih.gov/17515476/?from_single_result=Circulation+2007\%3B+115\%3A+2595-2598 (consultado el 10 de junio 2020).

4.- MALIK M, HNATKOVA K, NOVOTNY T, SCHMIDT G. Subject-specific profiles of QT/RR hysteresis. Am J Physiol Heart Circ Physiol. 2008; 295 (6): H2356 - 63. Disponible en https://pubmed.ncbi.nlm.nih.gov/18849333/ (consultado el 10 de junio 2020).

5.- BAZETT H. An analysis of the time-relations of electrocardiograms. Heart. 1920; 353 - 370. Disponible en https://onlinelibrary.wiley.com/doi/epdf/10.1111/j.1542-474X.1997. tb00325.x (consultado el 10 de junio 2020).

6.- SREDNIAWA B, MUSIALIK-LYDKA A, JARSKI P, SLIWINSKA A, KALARUS Z. Methods of assessment and clinical relevance of QT dynamics. Indian Pacing Electrophysiol J. 2005 Jul 1; 5 (3): 221 - 232. Disponible en https://pubmed.ncbi.nlm.nih.gov/16943870/?from_single_result=Methods+of+assessment+and+clinical+relevance+of+QT+dynamics.+Indian+Pacing+Electrophysiol (consultado el 10 de junio 2020). esta probabilidad alcanza al 100\%. Con base en lo expuesto y a la ausencia de estudios anteriores, dejamos disponible esta herramienta a toda la comunidad científica. A futuro será útil realizar un estudio prospectivo para establecer la utilidad y los valores de corte de esta razón, en una población de alto riesgo.

7.- PRIORI S, SCHWARTZ P, NAPOLITANO C, BLOISE R, RONCHETTI E, GRILLO $\mathrm{M}$, et al. Risk stratification in the long-QT syndrome. N Engl J Med. 2003 May 8; 348 (19):1866 - 1874. Disponible en https://pubmed.ncbi.nlm. nih.gov/12736279/?from_term=Risk+stratification+in+the+long-QT+syndrome (consultado el 10 de junio 2020).

8.- PRIORI S, BLOMSTRÖM-LUNDQVIST C, MAZZANTI A, BLOM N, BORGGREFE M, CAMM J, et al. 2015 ESC Guidelines for the management of patients with ventricular arrhythmias and the prevention of sudden cardiac death: The Task Force for the Management of Patients with Ventricular Arrhythmias and the Prevention of Sudden Cardiac Death of the European Society of Cardiology (ESC). Eur Heart J 2015 Nov 1; 36 (41): 2793 - 2867. Disponible en https://pubmed. ncbi.nlm.nih.gov/26320108/?from_single_result=Eur+Hear$\mathrm{t}+\mathrm{J} . \% 2 \mathrm{C}+36+\% 282015 \% 29 \% 2 \mathrm{C}+\mathrm{pp} .+2793-2867$ (consultado el 10 de junio 2020)

9.- COUMEL P. From provocation tests to clinical practice: invasive and non-invasive electrophysiology. Arch Mal Coeur Vaiss. 2003 May; 96 (4): 48 - 53. Disponible en https:// pubmed.ncbi.nlm.nih.gov/12852285/?from_single_result=From+provocation+tests+to+clinical+practice\%3A+invasive+and+non-invasive+electrophysiology (consultado el 10 de junio 2020).

10.-MASON J, RAMSETH D, CHANTER D, MOON T, GOODMAN D, MENDZELEVSKI B. Electrocardiographic reference ranges derived from 79.743 ambulatory subjects. J Electrocardiol. 2007; 40 (3): 228 - 234. Disponible en https:// pubmed.ncbi.nlm.nih.gov/17276451/ (consultado el 10 de junio 2020).

11.- PORTA-SÁNCHEZ A, GILBERT C, SPEARS D, AMIR E, CHAN J, NANTHAKUMAR K, et al. Incidence, Diagnosis, and Management of QT Prolongation Induced by Cancer Therapies: A Systematic Review. J Am Heart Assoc. 2017 Dec 7; 6 (12): e007724. Disponible en https://pubmed.ncbi.nlm.nih. gov/29217664/ (consultado el 10 de junio 2020). 